\title{
Usefulness of the $\mathrm{CHADS}_{2}$ and $\mathrm{R}_{2} \mathrm{CHADS}_{2}$ scores for prognostic stratification in patients with coronary artery disease
}

This article was published in the following Dove Press journal:

Clinical Interventions in Aging

\section{Yuerui Li \\ Juan Wang \\ Lyu Lv}

Cui Xu

Hongbin Liu

Department of Geriatric Cardiology,

Chinese PLA General Hospital, National Clinical Research Center for Geriatric Diseases, Beijing, China
Correspondence: Hongbin Liu Department of Geriatric Cardiology, Chinese PLA General Hospital, Fuxing Road 28, Haidian District, Beijing 100853, China Email liuhbin30I@I63.com
Objective: The current risk model for long-term prediction in coronary artery disease (CAD) is complicated, while a simple useful model is still lacking. We aim to investigate if $\mathrm{CHADS}_{2}$ and $\mathrm{R}_{2} \mathrm{CHADS}_{2}$ scores could predict long-term outcome for patients with CAD.

Patients and methods: We enrolled 3,700 patients with CAD between November 2010 and September 2014 at the Department of Cardiology from Chinese PLA General Hospital. The $\mathrm{CHADS}_{2}$ and $\mathrm{R}_{2} \mathrm{CHADS}_{2}$ scores were calculated. All cases were followed to track the incidence of composite end point consisting of cardiovascular (CV) death, myocardial infarction (MI), stroke, heart failure, and all-cause death.

Results: During a median 2.9-year follow-up, 443 patients experienced at least one element of the composite end point of CV death ( $n=168$ [4.6\%]), MI ( $n=59$ [1.6\%]), stroke ( $n=96$ [2.6\%]), heart failure $(n=101[2.8 \%])$, and all-cause death $(n=240[6.6 \%])$. Multivariate Cox regression analyses showed that the $\mathrm{CHADS}_{2}$ score (hazard ratio [HR]: $2.18,95 \% \mathrm{CI}$ : $2.00-2.38, p<0.0001$ ) and the $\mathrm{R}_{2} \mathrm{CHADS}_{2}$ score (HR: 1.93, 95\% CI: $1.83-2.04, p<0.0001$ ) were independently associated with composite outcome. Receiver-operating characteristic analysis showed that compared with the $\mathrm{CHADS}_{2}$ score, the $\mathrm{R}_{2} \mathrm{CHADS}_{2}$ score had better discrimination for the prediction of long-term combined outcome ( 0.772 vs $0.791, p=0.0013)$.

Conclusion: $\mathrm{CHADS}_{2}$ and $\mathrm{R}_{2} \mathrm{CHADS}_{2}$ scores provide a quick and useful tool in predicting long-term outcome for patients with CAD.

Keywords: $\mathrm{CHADS}_{2}$ score, $\mathrm{R}_{2} \mathrm{CHADS}_{2}$ score, coronary artery disease, prognosis, risk factors, renal function

\section{Introduction}

Coronary artery disease (CAD) is the leading cause of morbidity and mortality worldwide. ${ }^{1}$ Therefore, risk factor assessment and risk stratification of patients with CAD became important aspects of current research. However, the current risk model for long-term survival prediction for these patients was complicated, while a simple useful model was still lacking. ${ }^{2,3}$

The $\mathrm{CHADS}_{2}$ (congestive heart failure, hypertension, age, diabetes, and stroke/ transient ischemic attack) score, which assigns one point each for a history of congestive heart failure, hypertension, age $\geq 75$ years, diabetes mellitus (DM), and two points for prior stroke/transient ischemic attack (TIA), was used for embolic risk stratification and guidance in the treatment of anticoagulation for patients with non-valvular atrial fibrillation (AF). ${ }^{4}$ Recently, it was demonstrated that the $\mathrm{CHADS}_{2}$ score could predict clinical outcome in patients with acute myocardial infarction (MI), ${ }^{5}$ because the score included similar risk factors for poor prognosis. 
A new risk model, the $\mathrm{R}_{2} \mathrm{CHADS}_{2}$ (renal dysfunction, congestive heart failure, hypertension, age, diabetes, and stroke/ TIA) score, was proposed to be a powerful scoring scheme in predicting stroke or systemic embolism in AF patients. ${ }^{6}$ Renal dysfunction, the additional component of the $\mathrm{R}_{2} \mathrm{CHADS}_{2}$ score, was associated with worse clinical outcomes in CAD patients. ${ }^{7}$ Compared with the $\mathrm{CHADS}_{2}$ score, the $\mathrm{R}_{2} \mathrm{CHADS}_{2}$ score was believed to have better prognostic predictive value for stroke of AF patients. ${ }^{6}$ However, few studies have investigated the association of $\mathrm{R}_{2} \mathrm{CHADS}_{2}$ scores with long-term cardiovascular (CV) outcome in patients with CAD. Based on these experiences, we aimed to investigate if $\mathrm{CHADS}_{2}$ and $\mathrm{R}_{2} \mathrm{CHADS}_{2}$ scores could predict long-term outcome for patients with CAD.

\section{Patients and methods}

\section{Study populations}

We enrolled 3,700 patients with CAD between November 2010 and September 2014 at the Department of Cardiology from Chinese PLA General Hospital. Patients were included if they met the following criteria: age between 20 and 90 years, angiographic evidence of stenosis of $50 \%$ or greater in $\geq 1$ coronary vessel and hemodynamically stable. Patients were excluded if they had cardiogenic shock, severe valvular heart disease, myocarditis, severe anemia, active inflammatory disease, or cancer. The Medical Ethics Committee of PLA General Hospital approved the research protocol. All participants provided written informed consent.

\section{Procedures}

Demographic characteristics including age, sex, history of DM, hypertension, current cigarette smoking, chronic heart failure, previous ischemic stroke or TIA, medical history of percutaneous coronary intervention and/or coronary artery bypass grafting, and biochemical and echocardiographic examination were obtained from the hospital records. Hypertension was defined as repeated measurements of systolic blood pressure $\geq 140 \mathrm{mmHg}$, diastolic blood pressure $\geq 90$ $\mathrm{mmHg}$, or chronic treatment with antihypertensive medications. Diabetes was defined based on hospital records, hemoglobin A $1 \mathrm{c} \geq 7.0 \%$, or the use of antidiabetic medications. Congestive heart failure was defined as left ventricular ejection fraction $<40 \%$. Data on prior stroke, TIA, AF, and chronic obstructive pulmonary disease (COPD) were collected from the hospital records. Smoking was defined as smoking 10 cigarettes a day for at least 1 year without quitting. Family history was defined as the presence of heart disease or sudden cardiac death in a male first-degree relative aged $<55$ years or in a female first-degree relative aged $<65$ years.

\section{Calculation of $\mathrm{CHADS}_{2}$ and $\mathrm{R}_{2} \mathrm{CHADS}_{2}$ scores}

Thereafter, two experienced cardiologists, without knowledge of the patients' prognosis, calculated the $\mathrm{CHADS}_{2}$ and $\mathrm{R}_{2} \mathrm{CHADS}_{2}$ scores. The $\mathrm{CHADS}_{2}$ nomenclature represents congestive heart failure (C), HT (H), age (A), DM (D), and stroke (S). The $\mathrm{CHADS}_{2}$ score was calculated by assigning one point each for the presence of congestive heart failure, HT, and DM and by assigning two points for a history of stroke or TIA. The glomerular filtration rate (GFR) was calculated using the Chronic Kidney Disease Epidemiology (CKD-EPI) equation:

$$
\begin{aligned}
\text { GFR }= & 141 \times \min \left(\frac{\mathrm{Scr}}{\mathrm{k}, 1}\right)^{\mathrm{a}} \times \max \left(\frac{\mathrm{Scr}}{\mathrm{k}, 1}\right)^{-1.209} \\
& \times 0.993^{\text {Age }} \times[1.018 \text { if female }] \times[1.159 \text { if black }]
\end{aligned}
$$

The CKD-EPI equation was suggested to offer a more precise assessment of glomerular filtration, as compared to previous equations. ${ }^{8}$ The $\mathrm{R}_{2} \mathrm{CHADS}_{2}$ score was derived by incorporating the components of the $\mathrm{CHADS}_{2}$ score and awarding two points for renal dysfunction, defined as a GFR $<60 \mathrm{~mL} / \mathrm{min} /$ $1.73 \mathrm{~m}^{2}$. Scr is serum creatinine, $\mathrm{k}$ is 0.7 for female and 0.9 for male, a is -0.329 for female and -0.411 for male.

\section{Clinical outcome}

Follow-up data were collected by trained research coordinators through telephone interview and hospital records. We selected a composite end point consisting of MI, stroke, heart failure, and all-cause death. MI was defined as a clinical sign of infarction with recurrent chest pain and/or development of new electrocardiogram changes together with a rise of creatine kinase-MB or troponin- $\mathrm{T}$ measured following the chest pain. Stroke was defined as a new neurologic deficit, which could not be explained by other causes and with at least one image test (computed tomography or magnetic resonance imaging) compatible with the diagnosis, as well as confirmation from a neurologist. Heart failure was defined as hospitalization for signs and symptoms involving at least two of the following: orthopnea, paroxysmal nocturnal dyspnea, elevated jugular venous pressure, pulmonary rales, third heart sound, and pulmonary edema on radiography. Supportive documentation of reduced cardiac output and elevated pulmonary capillary wedge pressure was assessed when available. All-cause death included CV death and death caused by other reasons. CV death was defined as documentation of diagnoses involving ischemic heart disease, acute coronary syndrome (ACS), heart failure, 
cerebrovascular disease, arrhythmia, great vessel or peripheral vascular disease, valvular heart disease, or sudden death because of an unknown but presumed CV cause in high-risk patients. All patients were followed until they either reached the study end point or the end of study follow-up.

\section{Statistical analysis}

Descriptive statistics were performed for demographic and biomarker variables. The baseline data were summarized numerically as mean and SD and proportions for categorical variables. Differences between continuous values were assessed using ANOVA test for normally distributed variables. Differences between nominal variables were compared using the $\chi^{2}$ test. Cox proportional hazards models were used to identify the predictors of composite end point, $\mathrm{CV}$ death and all-cause mortality. Associations are reported as hazard ratios (HRs) with 95\% CIs. Kaplan-Meier curves were used to illustrate event rates for each risk level defined by score. A log-rank test was used to compare the survival curves among different patient groups. The receiver-operating characteristic curve was also used to demonstrate the sensitivity and specificity of the $\mathrm{CHADS}_{2}$ score and the $\mathrm{R}_{2} \mathrm{CHADS}_{2}$ score and their cutoff values for predicting clinical events. The area under the curve (AUC) comparison of these scoring systems was performed using the Delong method. A $p$-value of 0.05 was considered significant, and all tests were two tailed. Data were analyzed with SPSS software (version 19.0; IBM Corporation, Armonk, NY, USA) and MedCalc Statistical Software version 12.2 (MedCalc Software bvba, Ostend, Belgium).

\section{Results \\ Patient's characteristics}

After excluding patients with loss of follow-up $(n=55)$ or missing data required for the calculation of $\mathrm{CHADS}_{2}$ and $\mathrm{R}_{2} \mathrm{CHADS}_{2}$ scores $(\mathrm{n}=12)$, the remaining 3,633 subjects were the subjects of this secondary data analysis. The follow-up rate was $98.5 \%$. The median age was $61.5 \pm 11.7$ years, and subjects comprised 2,625 (72.3\%) men and 1,008 (27.7\%) women. The $\mathrm{CHADS}_{2}$ score ranged from 0 to 6 , with a mean $\pm \mathrm{SD}$ of $1.3 \pm 1.0$ and a median of $1(0-2)$, while the $\mathrm{R}_{2} \mathrm{CHADS}_{2}$ score ranged from 0 to 7 , with a mean \pm SD of $1.6 \pm 1.3$ and a median of $1(0-2)$. Baseline clinical characteristics according to tertiles of $\mathrm{R}_{2} \mathrm{CHADS}_{2}$ score are presented in Table 1. Subjects in the highest tertiles were older and more likely to have a history of hypertension, stroke, DM,

Table I Baseline clinical characteristics of patients

\begin{tabular}{|c|c|c|c|c|c|}
\hline Characteristics & $\begin{array}{l}\text { Overall } \\
\mathbf{N}=3,633\end{array}$ & $\begin{array}{l}0-2 \\
n=2,970\end{array}$ & $\begin{array}{l}3-5 \\
n=610\end{array}$ & $\begin{array}{l}6-8 \\
n=53\end{array}$ & $p$-value \\
\hline Age, years & $61.5 \pm 11.7$ & $59.6 \pm 10.7$ & $69.9 \pm 12.0$ & $73.2 \pm 12.4$ & $<0.001$ \\
\hline Male, n (\%) & $2,625(72.3)$ & $2,211(74.4)$ & $378(62.0)$ & $36(67.9)$ & $<0.001$ \\
\hline Smoking, n (\%) & I,09| (30.0) & 465 (15.7) & 103 (16.9) & $9(17.0)$ & $<0.001$ \\
\hline Previous stroke, n (\%) & $349(9.6)$ & $245(8.2)$ & $93(15.2)$ & II (20.8) & $<0.001$ \\
\hline Previous MI, n (\%) & 447 (12.3) & 329 (II.I) & 104 (I 7.0) & $14(26.4)$ & $<0.001$ \\
\hline Hypertension, n (\%) & $2,373(65.3)$ & I,79I (60.3) & $533(87.4)$ & $49(92.5)$ & $<0.001$ \\
\hline Hyperlipidemia, n (\%) & $\mathrm{I}, 126(3 \mathrm{I} .0)$ & $961(32.4)$ & 158 (25.9) & $7(13.2)$ & $<0.001$ \\
\hline Diabetes, n (\%) & $\mathrm{I}, \mathrm{I} 63(32.0)$ & $794(26.7)$ & $329(53.9)$ & $40(75.5)$ & $<0.001$ \\
\hline COPD, n (\%) & $85(2.3)$ & $50(1.7)$ & $30(4.9)$ & $5(9.4)$ & $<0.001$ \\
\hline$A F, n(\%)$ & $106(2.9)$ & $66(2.2)$ & $35(5.7)$ & $5(9.4)$ & $<0.001$ \\
\hline Previous PCl, n (\%) & 903 (24.9) & $702(23.6)$ & 176 (28.9) & $25(47.2)$ & $<0.001$ \\
\hline Previous CABG, n (\%) & $106(2.9)$ & $73(2.5)$ & $32(5.2)$ & I (I.9) & $<0.001$ \\
\hline $\mathrm{SBP}, \mathrm{mmHg}$ & $135.2 \pm 22.0$ & $134.3 \pm 21.9$ & $139.0 \pm 22.3$ & $142.8 \pm 23.0$ & $<0.001$ \\
\hline $\mathrm{DBP}, \mathrm{mmHg}$ & $75.8 \pm 16.6$ & $76.2 \pm 17.3$ & $74.0 \pm 12.6$ & $72.3 \pm 13.8$ & 0.004 \\
\hline $\mathrm{BMI}, \mathrm{kg} / \mathrm{m}^{2}$ & $25.6 \pm 3.4$ & $25.7 \pm 3.4$ & $25.1 \pm 3.6$ & $25.9 \pm 3.3$ & $<0.001$ \\
\hline $\mathrm{ABI}$ & $1.1 \pm 0.2$ & $\mathrm{I} . \mathrm{I} \pm 0.2$ & $\mathrm{I} .1 \pm 0.2$ & $0.9 \pm 0.3$ & 0.087 \\
\hline LVEF, \% & $56.5 \pm 8.4$ & $57.5 \pm 7.4$ & $52.3 \pm 10.5$ & $44.5 \pm 9.4$ & $<0.001$ \\
\hline $\mathrm{TC}, \mathrm{mg} / \mathrm{dL}$ & $4.0 \pm 1.1$ & $4.0 \pm 1.1$ & $4.0 \pm 1.1$ & $3.8 \pm 1.3$ & 0.209 \\
\hline $\mathrm{TG}, \mathrm{mg} / \mathrm{dL}$ & $1.6 \pm 1.0$ & $1.6 \pm 0.9$ & $1.6 \pm 1.0$ & $1.5 \pm 0.8$ & 0.384 \\
\hline HDL-C, mg/dL & $I . I \pm 0.3$ & $I .1 \pm 0.3$ & $I . I \pm 0.3$ & $1.0 \pm 0.3$ & 0.596 \\
\hline LDL-C, mg/dL & $2.4 \pm 0.9$ & $2.4 \pm 0.9$ & $2.4 \pm 0.9$ & $2.2 \pm 1.0$ & 0.159 \\
\hline Glucose, mg/dL & $7.0 \pm 4.8$ & $6.8 \pm 4.5$ & $7.8 \pm 5.9$ & $7.9 \pm 3.5$ & $<0.001$ \\
\hline Creatinine, $\mu \mathrm{mol} / \mathrm{L}$ & $86.1 \pm 67.9$ & 76. $I \pm 34.1$ & $128.7 \pm 132.6$ & $|56.3 \pm| 3||$. & $<0.001$ \\
\hline Multivessel disease, n (\%) & $2,229(61.4)$ & $\mathrm{I}, 807(60.8)$ & 390 (63.9) & $32(60.4)$ & 0.356 \\
\hline
\end{tabular}


Table I (Continued)

\begin{tabular}{|c|c|c|c|c|c|}
\hline \multirow[t]{2}{*}{ Characteristics } & Overall & $0-2$ & $3-5$ & $6-8$ & \multirow[t]{2}{*}{$p$-value } \\
\hline & $\mathrm{N}=3,633$ & $n=2,970$ & $n=610$ & $n=53$ & \\
\hline \multicolumn{6}{|l|}{ CAD type, n (\%) } \\
\hline SA & $907(25.0)$ & $786(26.5)$ & $113(18.5)$ & $8(15.1)$ & $<0.001$ \\
\hline UA & $2,297(63.2)$ & $1,857(62.5)$ & $405(66.4)$ & $35(66.0)$ & \\
\hline STEMI & $125(3.4)$ & $88(3.0)$ & $35(5.7)$ & $2(3.8)$ & \\
\hline NSTEMI & $304(8.4)$ & $239(8.0)$ & $57(9.3)$ & $8(15.1)$ & \\
\hline \multicolumn{6}{|l|}{ Medication, n (\%) } \\
\hline Aspirin & 3,398 (93.5) & $2,825(95.1)$ & $526(86.2)$ & 47 (88.7) & $<0.001$ \\
\hline Clopidogrel & $2,979(82.0)$ & $2,44 \mid(82.2)$ & $495(81.3)$ & 43 (8I.I) & 0.856 \\
\hline ACEI & $1,540(42.4)$ & $1,199(40.4)$ & $309(50.7)$ & $32(60.4)$ & $<0.001$ \\
\hline Statin & $3,431(94.5)$ & $2,830(95.3)$ & 555 (9I.I) & $46(86.8)$ & $<0.001$ \\
\hline Beta blocker & $2,627(72.5)$ & $2,134(72.0)$ & 455 (74.7) & $38(71.7)$ & 0.403 \\
\hline
\end{tabular}

Notes: Data are presented as mean \pm SD or $\mathrm{n}(\%) . \mathrm{R}_{2} \mathrm{CHADS}_{2}$, renal dysfunction, congestive heart failure, hypertension, age, diabetes, and stroke/transient ischemic attack. Abbreviations: $\mathrm{ABI}$, ankle brachial index; $\mathrm{ACEl}$, angiotensin converting enzyme inhibitor; $\mathrm{AF}$, atrial fibrillation; $\mathrm{BMI}$, body mass index; $\mathrm{CABG}$, coronary artery bypass grafting; CAD, coronary artery disease; COPD, chronic obstructive pulmonary disease; DBP, diastolic blood pressure; HDL-C, high-density lipoprotein cholesterol; LDL-C, lowdensity lipoprotein cholesterol; LVEF, left ventricular ejection fraction; MI, myocardial infarction; NSTEMI, non-ST-segment elevation myocardial infarction; PCI, percutaneous coronary intervention; SA, stable angina; SBP, systolic blood pressure; STEMI, ST-segment elevation myocardial infarction; TC, total cholesterol; TG, triglycerides; UA, unstable angina.

and MI. Demographic parameters such as systolic blood pressure, glucose, and creatinine were found to be significantly higher in patients with high $\mathrm{R}_{2} \mathrm{CHADS}_{2}$ score.

Over a median follow-up of 2.9 years, 443 patients experienced at least one element of the composite end point of $\mathrm{CV}$ death $(\mathrm{n}=168$ [4.6\%]), MI ( $\mathrm{n}=59$ [1.6\%]), stroke $(n=96[2.6 \%])$, heart failure $(n=101[2.8 \%])$, and all-cause death $(\mathrm{n}=240[6.6 \%])$. Kaplan-Meier plots showed that rates of composite outcome increased with increasing $\mathrm{CHADS}_{2}$ and $\mathrm{R}_{2} \mathrm{CHADS}_{2}$ scores (Figures 1 and 2). In terms of all-cause death and CV death, similar results were observed.

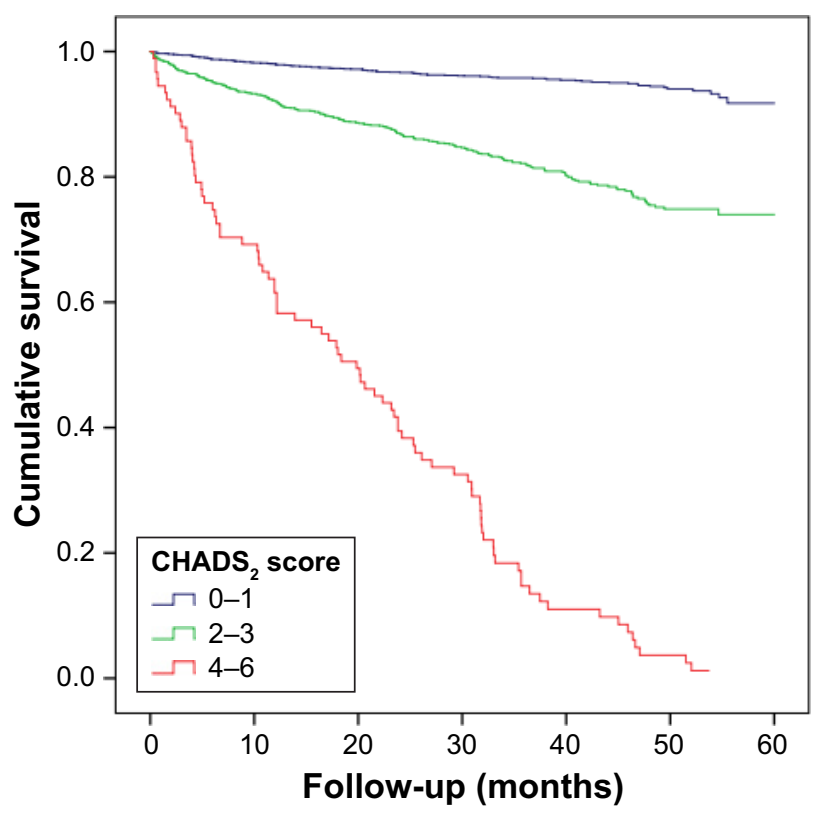

Figure I Event-free survival curve for patients according to the $\mathrm{CHADS}_{2}$ score. Note: $\mathrm{CHADS}_{2}$, congestive heart failure, hypertension, age, diabetes, and stroke/transient ischemic attack.

\section{Predictors of combined outcomes}

Using two separate multivariate Cox regression analyses (model 1 was adjusted for sex, smoking, body mass index, hyperlipidemia, GFR, total cholesterol, triglycerides, lowdensity lipoprotein cholesterol and high-density lipoprotein cholesterol, medication at discharge; model 2 was adjusted for the aforementioned covariates except GFR), the $\mathrm{CHADS}_{2}$ and $\mathrm{R}_{2} \mathrm{CHADS}_{2}$ scores were strongly associated with the composite end point (Table 2).

Then, we separately evaluated the prognostic value of the two scores in patients with stable CAD and ACS. After adjustment for additional covariates (model 1 for $\mathrm{CHADS}_{2}$

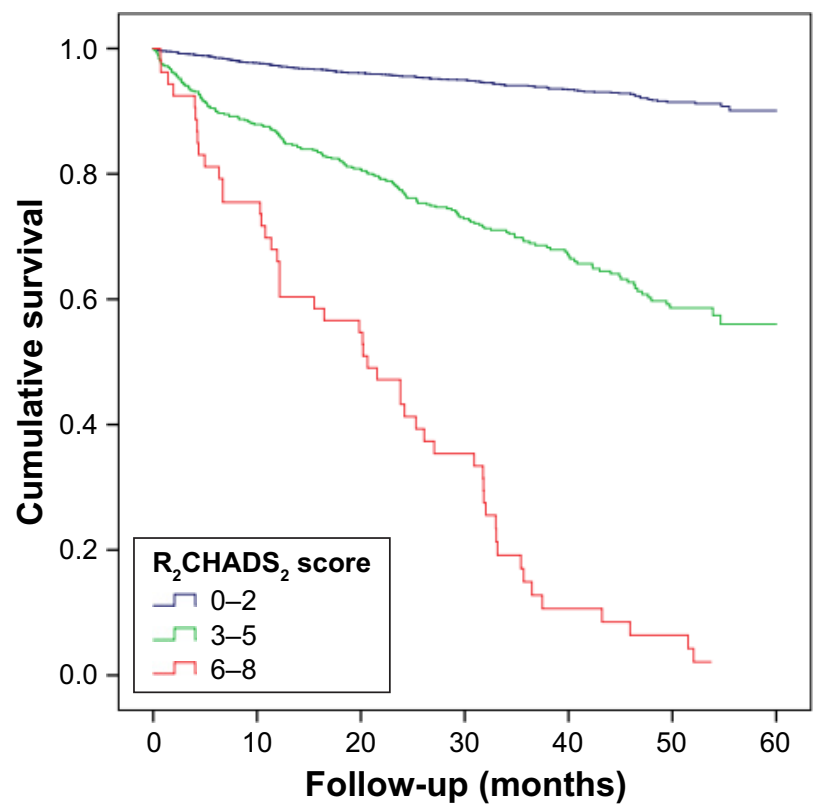

Figure 2 Event-free survival curve for patients according to the $\mathrm{R}_{2} \mathrm{CHADS}_{2}$ score. Note: $\mathrm{R}_{2} \mathrm{CHADS}_{2}$, renal dysfunction, congestive heart failure, hypertension, age, diabetes, and stroke/transient ischemic attack. 
Table 2 Predictors for composite outcome in the entire cohort

\begin{tabular}{|c|c|c|c|c|c|c|c|}
\hline \multirow[t]{2}{*}{ Variable } & \multicolumn{3}{|c|}{ Model I } & \multicolumn{4}{|c|}{ Model 2} \\
\hline & HR & $95 \% \mathrm{Cl}$ & $p$-value & HR & \multicolumn{2}{|l|}{$95 \% \mathrm{Cl}$} & $p$-value \\
\hline BMI & 0.9378 & $0.9116-0.9647$ & $<0.0001$ & 0.9400 & \multicolumn{2}{|l|}{$0.9131-0.9677$} & $<0.0001$ \\
\hline Hyperlipidemia & $0.580 \mathrm{I}$ & $0.4527-0.7433$ & $<0.0001$ & $0.574 I$ & \multicolumn{2}{|l|}{$0.4478-0.736 \mathrm{I}$} & $<0.0001$ \\
\hline TG & 0.8784 & $0.7794-0.9900$ & 0.0337 & 0.8791 & \multicolumn{2}{|l|}{$0.7796-0.9913$} & 0.0355 \\
\hline HDL-C & 0.6389 & $0.4546-0.8979$ & 0.0099 & 0.6444 & \multicolumn{2}{|l|}{$0.4586-0.9053$} & 0.0113 \\
\hline GFR & 0.9807 & $0.9769-0.9846$ & $<0.0001$ & & & & \\
\hline Aspirin & 0.7233 & $0.5372-0.9739$ & 0.0328 & 0.6852 & \multicolumn{2}{|l|}{$0.5159-0.9101$} & 0.0090 \\
\hline $\mathrm{CHADS}_{2}$ & 2.1848 & $2.0075-2.3777$ & $<0.0001$ & & & & \\
\hline $\mathrm{R}_{2} \mathrm{CHADS}_{2}$ & & & & 1.9299 & 1.8288 & 2.0365 & $<0.0001$ \\
\hline
\end{tabular}

Notes: Cox regression multivariate: model I was adjusted for sex, smoking, BMI, hyperlipidemia, GFR, TC, TG, LDL-C, HDL-C, and medication at discharge, while model 2 was adjusted for the aforementioned covariates except GFR. CHADS , congestive heart failure, hypertension, age, diabetes, and stroke/transient ischemic attack; $\mathrm{R}_{2} \mathrm{CHADS}_{2}$, renal dysfunction, congestive heart failure, hypertension, age, diabetes, and stroke/transient ischemic attack.

Abbreviations: BMI, body mass index; GFR, glomerular filtration rate; HDL-C, high-density lipoprotein cholesterol; HR, hazard ratio; LDL-C, low-density lipoprotein cholesterol; TC, total cholesterol; TG, triglycerides.

score, model 2 for $\mathrm{R}_{2} \mathrm{CHADS}_{2}$ score), the $\mathrm{CHADS}_{2}$ and $\mathrm{R}_{2} \mathrm{CHADS}_{2}$ scores were independent predictors of composite outcome, all-cause death and CV death, in both stable CAD and ACS patients (Table 3).

\section{Model performances}

Next, we performed receiver-operating characteristic analysis to determine the predictability of the CHADS 2 score and the $\mathrm{R}_{2} \mathrm{CHADS}_{2}$ score to the composite end point. The AUC was 0.772 (95\% CI: 0.758-0.785) for the $\mathrm{CHADS}_{2}$ score and 0.791 (95\% CI: 0.777-0.804) for the $\mathrm{R}_{2} \mathrm{CHADS}_{2}$ score. With a cutoff value of 2, the $\mathrm{CHADS}_{2}$ score had a sensitivity of $75.8 \%$ and a specificity of $66.7 \%$ to identify patients with poor clinical outcome. Meanwhile, with a cutoff value of 3 , the $\mathrm{R}_{2} \mathrm{CHADS}_{2}$ score had a sensitivity of $56.2 \%$ and a specificity of $87.0 \%$. Compared with the $\mathrm{CHADS}_{2}$ score, the $\mathrm{R}_{2} \mathrm{CHADS}_{2}$ score showed better discrimination for the prediction of long-term combined outcome ( 0.772 vs $0.791, p=0.0013$; Figure 3 ).

\section{Discussion}

In this study, we found that the $\mathrm{CHADS}_{2}$ score was a useful tool in risk stratification and long-term prognosis for patients with coronary heart disease. In particular, the new risk model, $\mathrm{R}_{2} \mathrm{CHADS}_{2}$ score, had better predictability than the CHADS 2 score of composite outcome for patients with CAD. Both $\mathrm{CHADS}_{2}$ and $\mathrm{R}_{2} \mathrm{CHADS}_{2}$ scores could predict long-term clinical outcome for patients with stable CAD and ACS.

The original purpose of the $\mathrm{CHADS}_{2}$ score was for risk stratification in stroke prevention of AF. Among several risk stratification indices, the $\mathrm{CHADS}_{2}$ score is the most commonly used because it is simple to calculate, well validated, and endorsed in practice guidelines. Later, the utility of the $\mathrm{CHADS}_{2}$ score in other CV fields attracted increasing attention. ${ }^{9}$ Tasolar et al enrolled 252 non-ST-segment elevation acute coronary syndrome (NSTE-ACS) patients and found that the $\mathrm{CHA}_{2} \mathrm{DS}_{2}$-VASc-HS score, which incorporated hyperlipidemia and smoking, was positively correlated with

Table 3 Predictors for clinical outcome in stable CAD and ACS patients

\begin{tabular}{|c|c|c|c|c|c|c|}
\hline & \multicolumn{3}{|c|}{ Stable CAD } & \multicolumn{3}{|l|}{ ACS } \\
\hline & HR & $95 \% \mathrm{Cl}$ & $p$-value & HR & $95 \% \mathrm{Cl}$ & $p$-value \\
\hline \multicolumn{7}{|c|}{ Composite outcome } \\
\hline $\mathrm{CHADS}_{2}^{\mathrm{a}}$ & 2.6671 & $2.1660-3.2843$ & $<0.0001$ & 2.1593 & $1.9830-2.3513$ & $<0.0001$ \\
\hline $\mathrm{R}_{2} \mathrm{CHADS}_{2}^{\mathrm{b}}$ & 2.0443 & $1.7664-2.3660$ & $<0.0001$ & 1.9184 & $1.8178-2.0246$ & $<0.0001$ \\
\hline \multicolumn{7}{|c|}{ All-cause death } \\
\hline $\mathrm{CHADS}_{2}^{\mathrm{a}}$ & 1.6533 & I.I768-2.3228 & 0.0037 & 1.5993 & $1.4 \mid 50-1.8077$ & $<0.0001$ \\
\hline $\mathrm{R}_{2} \mathrm{CHADS}_{2}^{\mathrm{b}}$ & 1.5429 & $1.2284-1.9379$ & $<0.0001$ & 1.6741 & $1.5519-1.8059$ & $<0.0001$ \\
\hline \multicolumn{7}{|c|}{ Cardiovascular death } \\
\hline $\mathrm{CHADS}_{2}^{\mathrm{a}}$ & 1.5574 & I.3347-1.8173 & $<0.0001$ & 1.5483 & I.1757-3.0364 & 0.0086 \\
\hline $\mathrm{R}_{2} \mathrm{CHADS}_{2}^{\mathrm{b}}$ & 1.6492 & $1.498 \mathrm{I}-1.8 \mathrm{I} 56$ & $<0.0001$ & 1.6282 & $1.4856-1.7846$ & $<0.000$ I \\
\hline
\end{tabular}

Notes: Cox regression multivariate: ${ }^{2}$ model I was adjusted for sex, smoking, BMI, hyperlipidemia, GFR, TC, TG, LDL-C, HDL-C, and medication at discharge, while ${ }^{\mathrm{b}}$ model 2 was adjusted for the aforementioned covariates except GFR. $\mathrm{CHADS}_{2}$, congestive heart failure, hypertension, age, diabetes, and stroke/transient ischemic attack; $\mathrm{R}_{2} \mathrm{CHADS}_{2}$, renal dysfunction, congestive heart failure, hypertension, age, diabetes, and stroke/transient ischemic attack.

Abbreviations: ACS, acute coronary syndrome; BMI, body mass index; CAD, coronary artery disease; GFR, glomerular filtration rate; HDL-C, high-density lipoprotein cholesterol; HR, hazard ratio; LDL-C, low-density lipoprotein cholesterol; TC, total cholesterol; TG, triglycerides. 


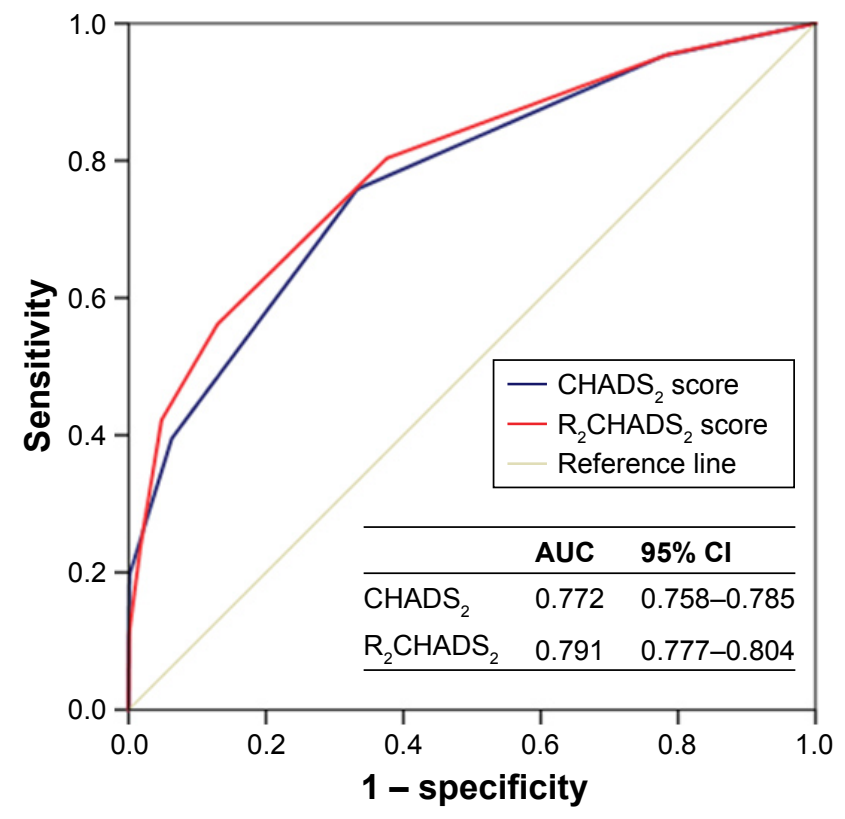

Figure 3 ROC curves for the $\mathrm{CHADS}_{2}$ and $\mathrm{R}_{2} \mathrm{CHADS}_{2}$ scores for predicting events. Note: $\mathrm{CHADS}_{2}$, congestive heart failure, hypertension, age, diabetes, and stroke/ transient ischemic attack; $\mathrm{R}_{2} \mathrm{CHADS}_{2}$, renal dysfunction, congestive heart failure, hypertension, age, diabetes, and stroke/transient ischemic attack.

Abbreviations: AUC, area under the curve; ROC, receiver operating characteristic.

the severity and complexity of CAD. ${ }^{10}$ They also found that $\mathrm{CHA}_{2} \mathrm{DS}_{2}$-VASc-HS was comparable with other risk scores for the risk stratification of the in-hospital major adverse cardiac events of NSTE-ACS patients. Welles et al enrolled 916 patients with stable $\mathrm{CAD}$ and no $\mathrm{AF}$ and reported that the $\mathrm{CHADS}_{2}$ score was strongly predictive of ischemic stroke/ TIA (AUC: 0.65 ). ${ }^{11}$ Poci et al enrolled 2,335 participants with ACS and reported that long-term mortality was associated with the $\mathrm{CHADS}_{2}$ score (HR: $1.38,95 \%$ CI: $1.28-1.48$ ). ${ }^{12}$

In line with these findings, this study demonstrated that higher $\mathrm{CHADS}_{2}$ score was associated with higher risk of combined outcome, all-cause death, and $\mathrm{CV}$ death in patients with CAD. It is of great importance to assess the risk of CAD patients to provide appropriate medical treatment and reduce $\mathrm{CV}$ events and mortality. Thus, several risk prediction scoring systems, including various CAD risk factors, have been developed. ${ }^{2,13,14}$ However, simple and reliable tools to identify CAD patients' risks are needed for routine practice. CHADS $_{2}$ score itself is proved to be a reliable and convenient tool to predict outcome for CAD patients. In fact, it is reasonable to expand the role of $\mathrm{CHADS}_{2}$ score in CAD, since each component of the $\mathrm{CHADS}_{2}$ score is also a risk factor of coronary heart disease and stroke itself also causes disability, further contributing to mortality.

Renal function is a powerful risk factor for mortality in patients with AF. Thus, Piccini et al have made attempts to combine $\mathrm{CrCl}$ with the $\mathrm{CHADS}_{2}$ score and created $\mathrm{R}_{2} \mathrm{CHADS}_{2}$ score. ${ }^{6}$ The $\mathrm{CrCl}$ was calculated with the Cockcroft-Gault formula. In this study, when calculating GFR, we used the CKD-EPI equation instead of the Cockcroft-Gault formula; it was because a previous study showed that GFR-based scheme, $\mathrm{R}_{2}(\mathrm{GFR}) \mathrm{CHADS}_{2}$, provided a significant improvement of predictive ability for mortality risk in older patients with AF. ${ }^{15}$ In a recent study, Huang et al enrolled 3,295 subjects with $\mathrm{CAD}$ and found that $\mathrm{R}_{2} \mathrm{CHADS}_{2}$ had comparable predictive ability of mortality to the Global Registry of Acute Coronary Events score. ${ }^{16}$ Compared with the CHADS ${ }_{2}$ score (c-statistic $=0.61$ ), the $\mathrm{R}_{2} \mathrm{CHADS}_{2}$ (c-statistic $=0.66$, $p<0.05)$ score provides better discrimination for mortality. The results of this study suggested that the $\mathrm{R}_{2} \mathrm{CHADS}_{2}$ score could be used to predict composite events for patients with $\mathrm{CAD}$, and the AUC of the $\mathrm{R}_{2} \mathrm{CHADS}_{2}$ was statistically larger than of the $\mathrm{CHADS}_{2}$ score. The clinical utility of the $\mathrm{R}_{2} \mathrm{CHADS}_{2}$ score should be emphasized, for those with a $\mathrm{R}_{2} \mathrm{CHADS}_{2}$ score of $\geq 3$ had a rate of adverse events as high as $37.6 \%$. These results indicated that reduced renal function played a critical role in the prognosis of $\mathrm{CV}$ outcomes in patients with CAD. Several potential mechanisms may explain these findings. Patients with reduced renal function often have consequences such as anemia, volume overload, and oxidative stress, which contribute to the poor outcomes. ${ }^{17}$ In addition, it has been reported that impaired renal function causes decreased number of smooth muscle cells within the plaque, which may accelerate the formation of vulnerable plaque and increase the possibility of plaque disruption. ${ }^{18,19}$

To our knowledge, although the $\mathrm{CHADS}_{2}$ score has already been tested in a previous study, the prognostic role of the $\mathrm{R}_{2} \mathrm{CHADS}_{2}$ score in long-term composite outcome in patients with CAD has not been addressed before. This study suggests that the $\mathrm{R}_{2} \mathrm{CHADS}_{2}$ score may predict risk with reasonable efficacy for patients with stable CAD and ACS.

\section{Limitations}

This study has several limitations. First, detailed information about the complexity of coronary artery lesions, such as the SYNTAX score, total or non-total arterial revascularization, was not evaluated. These factors are associated with longterm $\mathrm{CV}$ outcomes. In addition, the results need to be further validated in multicenter trials.

\section{Conclusion}

The CHADS ${ }_{2}$ and $\mathrm{R}_{2} \mathrm{CHADS}_{2}$ scores can be used to estimate the risk of clinical adverse events in patients with CAD. These 
scoring systems could lead to optimization of therapy, which might reduce the risks of subsequent adverse events.

\section{Acknowledgment}

This study was supported by grants from the National Key Research Program of China (2017YFC0840100 and 2017YFC0840103) and the Central Health Care Fund (W2015ZD02).

\section{Disclosure}

The authors report no conflicts of interest in this work.

\section{References}

1. Townsend N, Wilson L, Bhatnagar P, Wickramasinghe K, Rayner M, Nichols M. Cardiovascular disease in Europe: epidemiological update 2016. Eur Heart J. 2016;37(42):3232-3245.

2. Tang EW, Wong CK, Herbison P. Global registry of acute coronary events (GRACE) hospital discharge risk score accurately predicts long-term mortality post acute coronary syndrome. Am Heart J. 2007; 153(1):29-35.

3. Antman EM, Cohen M, Bernink PJ, et al. The TIMI risk score for unstable angina/non-ST elevation MI: a method for prognostication and therapeutic decision making. JAMA. 2000;284(7):835-842.

4. Rietbrock S, Heeley E, Plumb J, van Staa T. Chronic atrial fibrillation: Incidence, prevalence, and prediction of stroke using the Congestive heart failure, Hypertension, Age $>75$, Diabetes mellitus, and prior Stroke or transient ischemic attack (CHADS2) risk stratification scheme. Am Heart J. 2008;156(1):57-64.

5. Huang SS, Chen YH, Chan WL, Huang PH, Chen JW, Lin SJ. Usefulness of the CHADS2 score for prognostic stratification of patients with acute myocardial infarction. Am J Cardiol. 2014;114(9):1309-1314.

6. Piccini JP, Stevens SR, Chang Y, et al; ROCKET AF Steering Committee and Investigators. Renal dysfunction as a predictor of stroke and systemic embolism in patients with nonvalvular atrial fibrillation: validation of the $\mathrm{R}(2) \mathrm{CHADS}(2)$ index in the ROCKET AF (Rivaroxaban Once-daily, oral, direct factor $\mathrm{Xa}$ inhibition Compared with vitamin $\mathrm{K}$ antagonism for prevention of stroke and Embolism Trial in Atrial Fibrillation) and ATRIA (AnTicoagulation and risk factors in atrial fibrillation) study cohorts. Circulation. 2013;127(2):224-232.

7. Yiu KH, de Graaf FR, Schuijf JD, et al. Prognostic value of renal dysfunction for the prediction of outcome versus results of computed tomographic coronary angiography. Am J Cardiol. 2011;108(7):968-972.
8. Du X, Hu B, Jiang L, et al. Implication of CKD-EPI equation to estimate glomerular filtration rate in Chinese patients with chronic kidney disease. Ren Fail. 2011;33(9):859-865.

9. Lau KK, Chan PH, Yiu KH, et al. Roles of the CHADS2 and CHA2DS2VASc scores in post-myocardial infarction patients: risk of new occurrence of atrial fibrillation and ischemic stroke. Cardiol J. 2014; 21(5):474-483.

10. Tasolar H, Cetin M, Balli M, et al. CHA2DS2-VASc-HS score in non-ST elevation acute coronary syndrome patients: assessment of coronary artery disease severity and complexity and comparison to other scoring systems in the prediction of in-hospital major adverse cardiovascular events. Anatol J Cardiol. 2016;16(10):742-748.

11. Welles CC, Whooley MA, Na B, Ganz P, Schiller NB, Turakhia MP. The CHADS2 score predicts ischemic stroke in the absence of atrial fibrillation among subjects with coronary heart disease: data from the Heart and Soul Study. Am Heart J. 2011;162(3):555-561.

12. Poci D, Hartford M, Karlsson T, Herlitz J, Edvardsson N, Caidahl K Role of the CHADS2 score in acute coronary syndromes: risk of subsequent death or stroke in patients with and without atrial fibrillation. Chest. 2012;141(6):1431-1440.

13. Morrow DA, Antman EM, Charlesworth A, et al. TIMI risk score for ST-elevation myocardial infarction: a convenient, bedside, clinical score for risk assessment at presentation: an intravenous nPA for treatment of infarcting myocardium early II trial substudy. Circulation. 2000; 102(17):2031-2037.

14. Chen SL, Han YL, Zhang YJ, et al. The anatomic- and clinical-based NERS (new risk stratification) score II to predict clinical outcomes after stenting unprotected left main coronary artery disease: results from a multicenter, prospective, registry study. JACC Cardiovasc Interv. 2013; 6(12):1233-1241.

15. Fu S, Zhou S, Luo L, Ye P. R2(GFR)CHADS2 and R2(GFR) CHA2DS2VASc schemes improved the performance of CHADS2 and CHA2DS2VASc scores in death risk stratification of Chinese older patients with atrial fibrillation. Clin Interv Aging. 2017;12:1233-1238.

16. Huang FY, Huang BT, Pu XB, et al. CHADS2, CHA2DS2-VASc and R2CHADS2 scores predict mortality in patients with coronary artery disease. Intern Emerg Med. 2017;12(4):479-486.

17. Foley RN, Parfrey PS, Sarnak MJ. Clinical epidemiology of cardiovascular disease in chronic renal disease. Am J Kidney Dis. 1998;32(5 Suppl 3):S112-S119.

18. Wada M, Ueda Y, Higo T, et al. Chronic kidney disease and coronary artery vulnerable plaques. Clin J Am Soc Nephrol. 2011;6(12) 2792-2798

19. Kockx MM, Herman AG. Apoptosis in atherogenesis: implications for plaque destabilization. Eur Heart J. 1998;19(Suppl G):G23-G28.
Clinical Interventions in Aging

\section{Publish your work in this journal}

Clinical Interventions in Aging is an international, peer-reviewed journal focusing on evidence-based reports on the value or lack thereof of treatments intended to prevent or delay the onset of maladaptive correlates of aging in human beings. This journal is indexed on PubMed Central, MedLine,

\section{Dovepress}

CAS, Scopus and the Elsevier Bibliographic databases. The manuscript management system is completely online and includes a very quick and fair peer-review system, which is all easy to use. Visit http://www.dovepress. com/testimonials.php to read real quotes from published authors. 